\title{
酸性体系中纳米镍对 2,4-二氯苯酚降解性能的研究
}

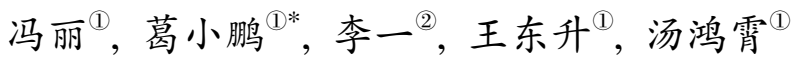 \\ (1) 中国科学院生态环境研究中心, 环境水质学国家重点实验室, 北京 100085; \\ (2) 中国钢研科技集团公司, 钢铁研究总院, 北京 100081 \\ * 联系人, E-mail: xpge@ @rcees.ac.cn
}

2011-02-14 收稿, 2011-05-04 接受

国家重点基础研究发展计划(2007CB407304)和国家水体污染控制与治理科技重大专项(2009ZX07209-004-2)资助项目

\begin{abstract}
摘要 纳米金属对有机氯化合物具有较好的脱氯降解效果, 但在反应过程中其表面易形成氧 化层覆盖, 并大量吸附目标反应物导致降解不完全, 而酸性体系可避免氧化层的形成. 对工业 羰基法生产的纳米镍超细粉进行了扫描电子显微镜(SEM)、透射电子显微镜(TEM)、X 射线衍 射(XRD)、能量色散谱仪(EDS)等微观结构观测与表征, 并就酸性体系中羰基纳米镍对 2,4-二氯 苯酚的脱氯降解性能进行了实验研究. 结果表明, 实验所用纳米镍的颗粒粒径为 $10 \sim 20 \mathrm{~nm}$, 表 面有一较薄的 $\mathrm{NiO}$ 层, 其存在将对 2,4-二氯苯酚的脱氯降解性能产生较大影响. 经酸洗活化处

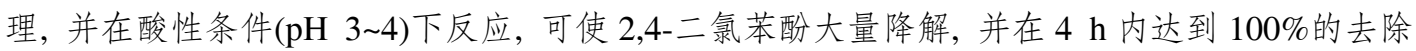
率. 反应溶液中可检测到 2-氯酚、4-氯酚、苯酚等降解产物的出现, 以苯酚为主. 反应过程中 因消耗质子需要定期补加酸量, 以维持体系适当的酸度, 酸不仅起到调节 $\mathrm{pH}$ 的作用, 更是作 为一种反应物参与反应, 从而极大地促进了脱氯降解反应进程. 酸性体系中纳米镍对氯代有机 物的脱氯降解反应为二级反应, 其反应速率随温度的升高而升高, 在 3 种不同温度 $(298,306$, $316 \mathrm{~K})$ 条件下, 其脱氯降解反应速率常数 $k$ 分别为 $0.02,0.2$ 和 $0.3(\mathrm{~g} \mathrm{~L} \mathrm{~h})^{-1}$.
\end{abstract}

\section{关键词}

纳米镍粉

$2,4-$ 二氯苯酚

吸附

降解

酸性体系
零价纳米金属因其粒径小、具有较大的比表面积 及表面能 ${ }^{[1,2]}$, 从而具有优越的吸附性能和较高的还 原活性 ${ }^{[3]}$, 成为一种有效的脱卤还原剂, 并在有机氯 废水等处理方面具有广阔的应用前景. 例如, 零价纳 米铁(nZVI) 以其特有的表面效应和小尺寸效应, 可 催化还原多种有机卤化物, 在处理氯代脂肪烃 ${ }^{[4,5]}$ 、氯 代芳香烃 ${ }^{[6]}$ 、氯代苯酚 ${ }^{[7]}$ 和有机氯农药 ${ }^{[8]}$, 甚至多溴 联苯醚 ${ }^{[9]}$ 等方面表现出显著的作用效果, 并将这些 难降解有机污染物转化为无毒或低毒的化合物, 近 年来开始广泛用于环境污染治理 ${ }^{[10,11]}$.

工业羰基热分解法是一种选择性很强的精炼高 纯金属粉末的特殊工艺技术. 它利用羰基金属化合 物的热离解来制取金属粉末, 主要用于金属铁、镍等 粉末的制备. 羰基法制取的金属粉末纯度较高, 具备 作为环境纳米材料的潜质. 侯春风等 ${ }^{[12]}$ 对羰基法生
产的纳米铁降解 2,4-二氯苯酚的性质进行了初步研 究, 结果表明羰基纳米铁粉对 2,4-二氯苯酚具有很好 的去除效果, 其中有较大一部分去除来自多孔性纳 米铁材料本身及其表面疏松氧化层的吸附作用. 然 而纳米铁颗粒强烈的吸附性能对于有机氯化合物的 去除有利有弊. 一方面, 在反应初期强大的吸附能力 促进了污染物的降解过程; 另一方面, 在反应后期, 由于纳米铁表面形成的疏松氧化层的吸附作用, 部 分污染物质并非完全降解，而是吸附在纳米铁颗粒 物表面上继续存在，从而有可能跟随纳米铁颗粒在 水体中迁移, 导致污染物的扩散. 由于纳米铁性质活 泼, 它在反应过程中氧化很快, 并生成大量的惰性反 应层吸附包裹目标产物. 有研究表明, 纳米金属反应 过程中形成的疏松氧化层对目标物的吸附作用是导 致多卤代化合物不完全降解的主要原因 ${ }^{[13]}$, 而有酸

英文版见: Feng L, Ge X P, Li Y, et al. Dechlorination of 2,4-dichlorophenol by nickel nanoparticles under the acidic conditions. Chinese Sci Bull, 2011, 56, doi: $10.1007 / \mathrm{s} 11434-011-4549-1$ 
存在时, 可抑制这种情况的发生.

另外, 纳米铁在空气中不稳定, 且很容易自燃 ${ }^{[14]}$; 在实际应用时, 纳米铁酸洗活化过程中造成的损失 也较大. 由于氧化、水解等现象的发生其对目标物反 应虽然剧烈，但难以持续，且不易把握。而作为铁的 同族元素, 镍的物理化学性质与铁有很多相似之处, 但远没有铁活泼, 氧化水解过程也相对缓慢, 并且在 羰基法生产工艺 ${ }^{[15]}$ 中其生成的氧化层厚度很薄. 羰 基法纳米镍的这些特点对实验操作和检测都有很大 益处. 本文以羰基纳米镍为研究对象, 试验了其在酸 性体系中对 2,4-二氯苯酚脱氯降解性能, 以期加深人 们对纳米金属的脱氯还原过程与机理的了解与认识, 并对纳米铁的性能与机理做出对比和补充.

\section{1 材料与方法}

(i ) 主要试剂与仪器. 主要试剂: 2,4-二氯苯 酚(分析纯, 国药集团化学试剂有限公司); 纳米镍超 细粉, 来自中国钢研科技集团公司钢铁研究总院, 由 羰基镍经加热分解, 在 $\mathrm{N}_{2}$ 保护气氛条件下气相沉积 而成; 甲醇(分析纯, Fisher 公司, 美国), 娃哈哈纯净 水(娃哈哈集团有限公司); 混纤微孔滤膜(孔径 $0.45 \mu \mathrm{m}$, 北京升河诚信膜科技发展中心). 主要仪器: 透射电 子显微镜(S-570, Hitachi 公司, 日本), D/Max-RC 型 $X$ 射线衍射光谱仪(Rigaku 公司，日本)，扫描电子显 微镜(S-3000N, Hitachi, 日本), EDS 能谱仪(EDAX, 美国)，ASAP-2000 型比表面及孔径分布测定仪 (Micromeritics 公司, 美国), 磁力搅拌器(90-4 恒温磁 力搅拌器, 上海振荣科学仪器有限公司), 恒温震荡 器(ZD-85A 气浴恒温振荡器), 高效液相色谱仪(1525 二元高压梯度洗脱, Waters, 美国).

(ii) 纳米镍的理化性能测试. 采用扫描电子显 微镜 (SEM) 和透射电子显微镜(TEM) 观测纳米镍的微 观结构形貌, 并利用 EDS 能谱仪进行纳米镍颗粒物 表面的元素成分分析. 对于 SEM-EDS 分析检测, 将 粉末样品直接在碳膜上制样后进行观测; 而对于 TEM 观测, 则先将纳米镍用乙醇溶剂超声分散, 然 后于碳支架膜上制样, 风干后进行观测; 以 $\mathrm{D} / \mathrm{Max}-\mathrm{RC}$ 型 $\mathrm{X}$ 射线衍射光谱仪分析纳米镍粉的物相组成, 衍 射条件为 $\mathrm{Cu}$ 靶, $40 \mathrm{kV}, 40 \mathrm{~mA}$, 采用连续扫描方式, 扫描范围为 $5 \sim 100^{\circ}$, 步宽 $0.033^{\circ}$; 采用 $\mathrm{N}_{2}$ 吸附法以 ASAP-2000 型比表面及孔径分布测定仪测其比表面 积及孔容孔径, 测试之前样品先在 $90^{\circ} \mathrm{C}$ 预处理 $1 \mathrm{~h}$,
然后在 $350^{\circ} \mathrm{C}$ 下处理 $3 \mathrm{~h}$ 后进行测定.

(iii) 未酸化纳米镍对 2,4-二氯苯酚的降解实验. 称取 $1.5 \mathrm{~g}$ 纳米镍粉于雉形瓶中, 用纯净水制成悬浮 液, 经超声分散 $20 \mathrm{~min}$ 后, 加人 $200 \mathrm{~mL} 5 \mathrm{mg} / \mathrm{L}$ 的 2 , 4-二氯苯酚溶液, 密封. 一定温度下置于恒温震荡器 上反应, 转速为 $200 \mathrm{r} / \mathrm{min}$, 在规定的时间取样, 并进 行固液分离(固液分离均使用 $0.45 \mu \mathrm{m}$ 膜过滤方法), 取上清液于高效液相色谱仪上检测 2,4-二氯苯酚的 去除降解与产物的生成情况, 并同时进行平行样品 与空白对照实验.

（iv）酸洗活化纳米镍在中性及酸性体系内对 2,4-二氯苯酚的降解实验. 经初步实验证实, 纳米 镍酸洗后损失不超过 $15 \%$. 因此, 在计算纳米镍的投 加量时, 酸洗活化后纳米镍的损失应考虑在内, 本实 验按 $15 \%$ 计算. 称取适量纳米镍粉于三角瓶中, 加人 体积比为 $1: 200$ 的硫酸溶液, 然后于磁力搅拌器上反 应 $1 \mathrm{~h}$, 反应完成后, 经纯水多次洗涤, 待 $\mathrm{pH}$ 接近中 性后, 定容, 此时悬浮液浓度为 $15 \mathrm{~g} / \mathrm{L}$.

使用前先进行超声分散 $20 \mathrm{~min}$, 然后移取 $100 \mathrm{~mL}$ 于锥形瓶中, 加人 $100 \mathrm{~mL} 10 \mathrm{mg} / \mathrm{L}$ 的 2,4-二氯苯酚溶 液, 密封. 一定温度下置于恒温震荡器上反应, 转速 为 $200 \mathrm{r} / \mathrm{min}$, 在规定时间取样, 过 $0.45 \mu \mathrm{m}$ 滤膜进行 固液分离后, 于高效液相色谱仪处检测 2,4-二氯苯酚 的去除降解与产物的生成情况. 对于活化后中性体 系的实验, 在纯水洗涤多次至中性后直接使用; 而对 于酸性体系, 则在反应过程中使用 $1: 200$ 的分析纯硫 酸调节 $\mathrm{pH}$, 使溶液酸度稳定在 $\mathrm{pH} 3 \sim 4$ 范围内.

高效液相色谱仪实验条件为: Waters C18 色谱柱, 流动相配比为甲醇: 水 $=70: 30(\mathrm{v} / \mathrm{v})$, 采用 Waters 2487 检测器, 检测波长为 $280 \mathrm{~nm}$, 手动进样.

(V) 纳米镍对 2,4-二氯苯酚的解吸以及对其降 解产物苯酚的吸附解吸实验。在上述未酸化纳米 镍、酸洗活化纳米镍中性及酸性等 3 种不同反应体系 中, 待吸附降解反应完成后, 对不同体系的样品进行 离心和固液分离, 并在得到的固体样品中重新加人 $200 \mathrm{~mL}$ 超纯水, 在相同条件下对反应后的纳米镍粉 进行解吸实验. 在规定时间取样, 并过 $0.45 \mu \mathrm{m}$ 滤膜 进行固液分离后, 于高效液相色谱仪处检测 2,4-二氯 苯酚反应物与降解产物的溶出情况.

同时, 初步实验还发现, 在中性体系的实验过程 中, 2, 4-二氯苯酚的主要降解产物一苯酚的出现并无 明显的规律性. 为此, 本实验还对纳米镍颗粒物在该 
实验条件下对苯酚的吸着及解吸行为进行了进一步 实验研究. 将经酸洗活化纳米镍与一定浓度的苯酚 溶液按上述静态吸附实验条件进行实验, 并在规定 时间取样, 分析其溶液中残余情况; 将达到吸附平衡 后的纳米镍样品, 重新悬浮到水体中进行解吸实验, 研究其溶出解吸情况.

\section{2 结果与讨论}

\section{1 纳米瀪的理化性能}

(i) 纳米镍粉的微观形貌与比表面积. 图 1 为 纳米镍颗粒物在不同分散状态及分辨率条件下的微 观结构形貌图像, 左侧(图 1(a))的 SEM 图片给出了纳 米镍团聚体颗粒的较低分辨率图像, 表明在自然分 散状态下纳米镍由无数细小的颗粒 (一次粒子)相互 团聚成尺度更大的微米级颗粒团簇. 这是由于纳米 颗粒比表面积大, 活性高, 倾向于团聚以稳定, 而且 纳米镍具有磁性, 从而更加易于团聚. 这些团簇粒子 内部存在大量结构缺陷, 其形状、方位等也存在差异, 粉体表面非活性吸附中心的数目较多. 由图 1(b)经乙 醇分散后的纳米镍高分辨 TEM 图像可知, 分散后的 纳米镍颗粒以较松散的链状结构聚集体存在, 单个 颗粒粒径范围为 10 20 nm. 使用氮吸附法测定纳米 镍的比表面积为 $32.40 \mathrm{~m}^{2} / \mathrm{g}$, 这一检测结果为纳米镍 颗粒在自然团聚状态下的比表面积, 而与相同工艺 制备的纳米铁粉相比, 其比表面积增加了近 4 倍.

(ii) 纳米镍颗粒的组成与结构. 为进一步了解 纳米镍的结构组成, 对纳米镍粉进行了 X 射线衍射 (XRD)与能量色散谱仪(EDS)的分析表征. 图 2 给出 了纳米镍的 XRD 谱图. 可以看出, 在 30 100 扫描衍 射角范围内, 纳米镍样品衍射峰所对应的 $2 \theta$ 角分别 为 $44.498^{\circ}, 51.877^{\circ}, 76.457^{\circ}, 93.106^{\circ}$. 根据布拉格

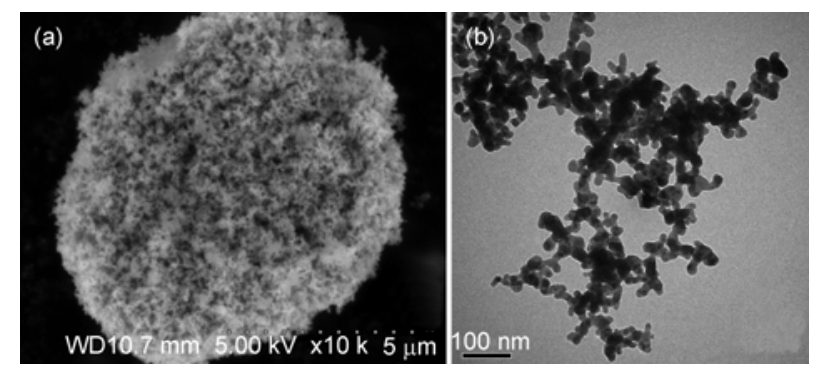

图 1 纳米镍的微观形貌图

(a) SEM 图片; (b) TEM 图片

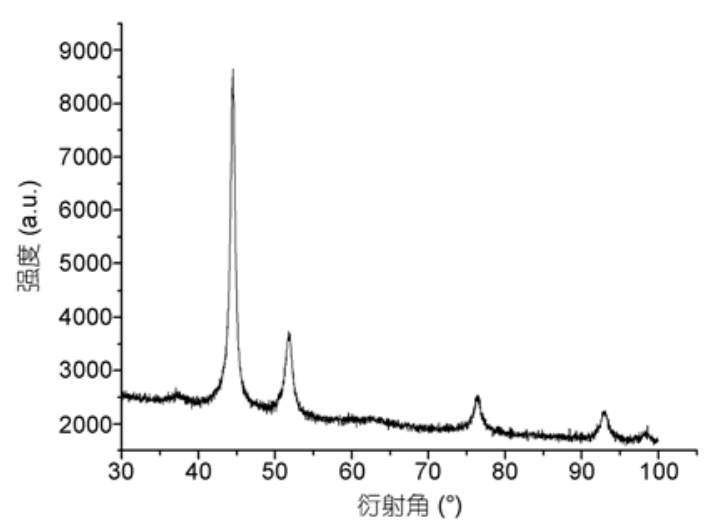

图 2 纳米镍的 XRD 谱图

(Bragg)方程 $2 d \sin \theta=n \lambda$, 其中 $d$ 为相邻平行晶面的 晶面间距, $\theta$ 为人射角, $\lambda$ 为人射波的波长, $n$ 为衍射级 数, 在此其值取为 1 , 可以得出相应的晶面间距 $d$ 分 别为: $2.034,1.760,1.245$ 和 $1.061 \AA$. 对照镍的标准卡 片分别对应于镍的 111(2.035 ̊)、200(1.762 ̊)、220 $(1.246 \AA)$ 和 $311(1.063 \AA)$ 晶面的衍射峰, 晶型为面 心立方结构 $(\mathrm{fcc})$. 另外, 在 $2 \theta$ 角为 $37.282^{\circ}$ 和 $63.563^{\circ}$ 处还发现有微弱的衍射峰, 经对照标准卡片知其为 镍的氧化物 $(\mathrm{NiO})$ 的衍射峰, 由于其强度很弱表明此 纳米镍粉末中氧化镍含量很少. 而根据纳米镍的制 备工艺, 该样品表面也应该含有镍的氧化物, 从而单 质镍纳米颗粒表面有很薄的氧化层包裹着, 在常温 下比较稳定.

根据谢乐公式 $d=K \lambda / \beta \cos \theta$, 通过最强衍射峰的 半峰宽(FWHM)可计算出金属镍纳米颗粒的尺寸. 其 中, $K$ 为 Scherrer 常数, 其值为 $0.89 ; d$ 为晶粒尺寸 $(\mathrm{nm}) ; \beta$ 为衍射线的本征加宽, 即经双线校正和仪器 因子校正得到的完全由于晶粒大小引起的衍射线加 宽, 通常用衍射峰极大值一半处的宽度表示. 在计算 $\beta$ 时, 换算为弧度 $(\mathrm{rad})$, 为扣除仪器宽化误差, 将半 峰宽减去 $0.08 ; \theta$ 为布拉格角 (半衍射角); $\lambda$ 为 $\mathrm{X}$ 射线 的波长, 其值为 $0.15405 \mathrm{~nm}$. 由此可计算出, $\mathrm{Ni}$ 粒径 为 $11.3085 \mathrm{~nm}$, 其结果与透射电镜观测结果一致.

由表 1 纳米镍的 EDS 分析结果可以看出, 羰基 纳米镍中含有少量的碳和氧元素, 其质量百分比为 $\mathrm{C}: \mathrm{O}: \mathrm{Ni}=3.11: 4.40: 92.49$, 原子数比为 $\mathrm{C}: \mathrm{O}: \mathrm{Ni}=12.28$ ： 13.04:74.68. 氧元素的引人是由于纳米镍的超高活性, 工艺过程中钝化而引人的, 而碳元素的存在则是由 于其制备工艺采取羰基热分解气相沉积而成, 多孔 性纳米金属粉末结构导致存在一定量的碳含量残余. 
表 1 纳米镍的能量色散(EDS)分析结果

\begin{tabular}{ccc}
\hline 元素 & 质量分数 $(\%)$ & 原子分数 $(\%)$ \\
\hline $\mathrm{C}$ & 3.11 & 12.28 \\
$\mathrm{O}$ & 4.40 & 13.04 \\
$\mathrm{Ni}$ & 92.49 & 74.68 \\
\hline
\end{tabular}

\section{2 不同介质条件下纳米镍对 2,4-二氯苯酚的降 解效果比较}

本实验中选取了 3 种体系对纳米镍粉的脱氯作 用进行了考察. 一是将工业纳米镍粉经纯净水分散 后, 直接投加氯酚溶液进行脱氯反应; 二是将工业纳 米镍粉首先进行酸洗活化后再投加, 由于酸洗后的
纳米镍经超纯水洗涤干净, 整个体系呈中性偏碱的 状态，接下来称其为中性体系; 三是在酸洗活化后的 镍与氯酚的反应体系中持续加酸保持在较低 $\mathrm{pH}$ 下进 行反应.

图 3 给出了等量的纳米镍在不同的处理方法或 体系中对 2,4-二氯苯酚的浓度和去除率变化情况. 其 中图 3(a)和图 3(b) 是未酸洗活化的纳米镍去除 2,4-二 氯苯酚的浓度和去除率变化情况, 图 3(c)和图 3(d)是 酸洗活化后中性和酸性体系中去除 2,4-二氯苯酚的 浓度和去除率变化情况. 观察对比可知, 未经酸洗的 纳米镍粉在反应开始时有一段迟滞期, 图 3(a) 和图 3(b)中将反应的前 $5 \mathrm{~h}$ 放大图表示, 可看出反应的波 动, 这是由于未酸洗活化的纳米镍粉表面存在致密
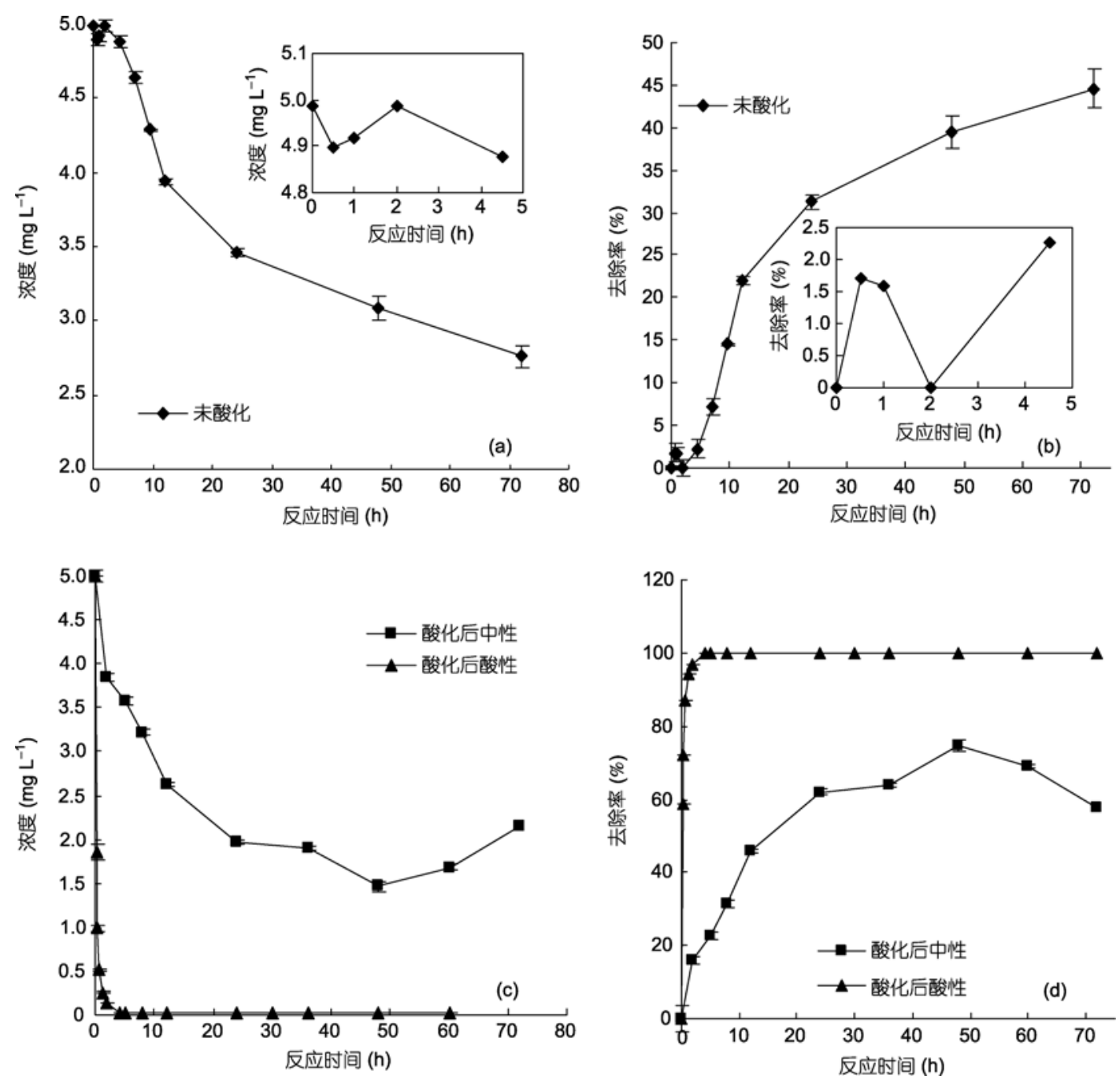

图 3 不同介质条件下纳米镍粉对 2, 4-二氯苯酚的吸附与脱氯降解过程比较

(a) 未酸化体系的浓度变化; (b) 未酸化体系的去除率变化; (c) 酸化后两种体系的浓度变化; (d) 酸化后两种体系的去除率变化 
的氧化层, 且呈疏水性, 加人反应溶液后需要经历一 个逐渐润湿的传质平衡过程, 约 $5 \mathrm{~h}$ 后才出现明显的 去除作用，其对 2,4-二氯苯酚的去除率最高可达 $40 \%$; 由图 3(c) 和图 3(d) 可知, 酸洗后的中性体系对 2,4-二 氯苯酚的去除率可以达到 $70 \%$ 以上, 但随着接触反 应时间的延长, 去除率又呈现下降的趋势; 而且, 当 使用稀硫酸使体系在反应过程中保持酸性( $\mathrm{pH}$ 3 4) 时, 2,4-二氯酚的浓度得以迅速的降低, 并且达到最 高去除率后非常稳定，再无氯酚浓度波动的情况出现，

由于未酸洗体系和酸洗后中性体系中出现去除 率增大又减小的现象, 且反应过程中几乎没有检测 到脱氯降解产物出现, 表明 2,4-二氯酚未被完全降解, 只是吸附于纳米镍颗粒表面上. 于是在反应结束后, 对不同体系的样品进行离心达到固液分离, 并对反 应后的纳米镍颗粒物做进一步解吸实验. 结果显示, 上述反应体系的纳米镍果然释放出一定量的 2,4-二 氯酚, 从而验证了上述介质体系条件下, 纳米镍对 2 , 4-二氯酚未完全降解的推断结论.

具体解吸实验结果如图 4所示, 从图可以看出: 对于吸附后的纳米镍粉, 2,4-二氯苯酚从其上的解吸 有两个特点: 一是它并不会将全部吸附的镍量再次 释放出来, 而是仅仅释放出一部分; 二是在解吸试验 中, 2,4-二氯苯酚很快被释放出来并达到平衡, 随着 接触时间的延长, 其浓度不再有明显变化. 因为吸附 过程包括物理吸附和化学吸附两部分, 由实验现象 推断, 很快被释放出来的这一部分, 是通过物理作用 结合在纳米镍表面, 经解吸作用可以释放出来, 而未 释放出来的部分则是主要以化学吸附作用存在, 即 这部分 2,4-二氯苯酚虽然暂时没有被降解, 但通过化 学作用紧密结合于纳米镍氧化物的表面, 很难再释 放出来. 之前也有研究指出, 利用傅里叶红外分析表 明氯酚类化合物可通过配位化学作用吸附于氧化物 表面，并且无法通过水洗去除 ${ }^{[16]}$, 而在有酸存在时 则可达到完全去除. 图 4结果表明, 在酸性体系内 2,4-二氯苯酚可迅速被纳米镍吸附去除, 并且在解吸 过程中，2,4-二氯苯酚没有从纳米镍中释放出来，证 明 2,4-二氯苯酚在该反应体系中已经被完全降解.

综合三种体系的试验结果可知, 未经酸洗活化 的纳米镍粉由于表面有一层较薄氧化层, 活性较低, 其对 2,4-二氯苯酚的去除基本上来自吸附作用; 而酸 洗之后, 氧化层已被去除, 团聚现象大大改善, 比表 面积提高, 纳米镍活性增高, 吸附及降解能力都大大
提高. 在酸化后的中性体系中, 纳米镍对 2,4-二氯苯 酚的去除包括吸附和降解两种作用: 在反应开始的 阶段, 2,4-二氯酚首先被大量的纳米镍吸附, 并发生 脱氯降解，2,4-二氯酚的去除率明显提高. 但随着反 应的持续进行, 纳米镍表面开始氧化, 形成疏松的氧 化层, 体系内 $\mathrm{pH}$ 升高, 此时的去除效果主要来自吸 附作用, 脱氯降解作用变差. 接下来随着接触时间的 延长, 物理吸附于纳米镍表面的一部分 2,4-二氯酚解 吸下来, 总体表现出去除率下降. 而在酸性体系中的 纳米镍, 由于酸的存在, 氢离子来源稳定, $\mathrm{pH}$ 较低, 防止了氧化层的产生, 加速了镍的腐蚀, 降解速度提 高, $4 \mathrm{~h}$ 内可将 2,4-二氯酚完全去除, 并检测到有苯酚 等产物出现, 而且在解吸过程中没有再出现 2,4-二氯 酚, 说明此时去除效果主要来自脱氯降解.

之前有研究表明, 零价金属对氯代烃有机物的 去除, 其吸附过程起主要作用 ${ }^{[17,18]}$. 这些研究所采用 的金属材料主要是生铁, 其中含有石墨碳, 而正是其 中的碳质成份起到了主要的吸附作用 ${ }^{[19,20]}$. 在本实 验中, 羰基法纳米金属由于其生产工艺的原因, 其中 也含有碳, 其作用机制与上述研究有些类似, 即在中 性体系内纳米镍对 2,4-二氯苯酚的去除主要来自其 吸附作用. 在中性体系中, 新生的氧化物、氢氧化物 将包裹在纳米镍颗粒物表面. 受传质过程的影响, 从 而阻碍了未反应的目标物进人到纳米镍颗粒内部发 生继续反应或降解产物到溶液中的进一步释放过程; 而在酸性体系中氧化物、氢氧化物的生成将受到抑制,

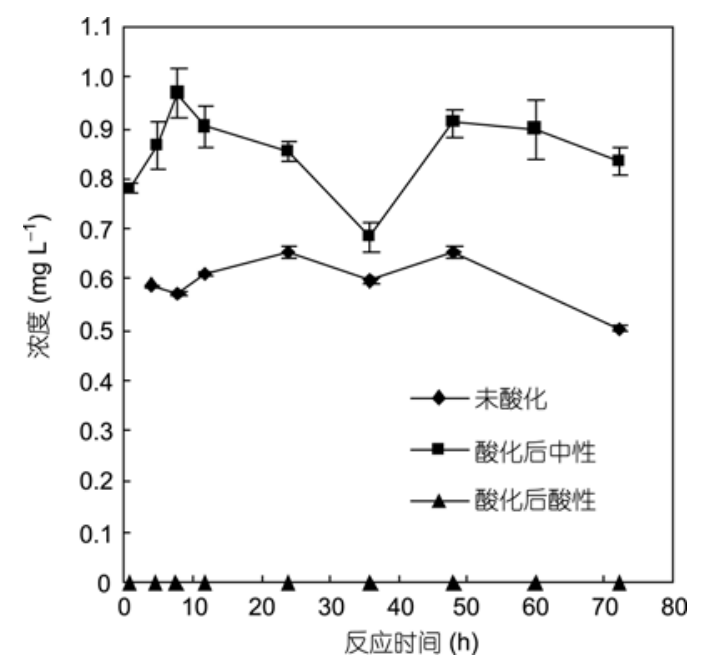

图 42,4 -二氯苯酚从不同体系纳米镍粉吸附产物 的解吸情况 
初期吸附在纳米镍上的氯酚可进一步脱氯降解, 因 而本实验采用的酸性体系可极大地提高 2,4-二氯苯 酚的脱氯降解效率.

\section{3 酸性体系中不同温度下纳米镍对 2,4-二氯苯 酚的吸附与降解性能}

2,4-二氯苯酚的脱氯反应为固液两相反应, 其脱 氯降解过程涉及 3 个步骤: (1) 溶液中 2,4 -二氯苯酚 向固体( $\mathrm{Ni}$ 金属)表面的质量传递; (2) 2,4-二氯苯酚吸 附在 $\mathrm{Ni}$ 表面并发生脱氯降解反应; (3) 2,4-二氯苯酚 的脱氯产物脱附并向主体溶液的扩散. 在本实验条 件下, 由于震荡速度很快, 反应体系中不存在质量传 递控制; 同时, 2,4-二氯苯酚在纳米镍表面上的吸附 也很容易发生. 在酸性体系中, 整个脱氯过程的控制
步骤应该是 2,4 -二氯苯酚和 $\mathrm{H}^{+}$在 $\mathrm{Ni}$ 表面上的化学反 应. 经实验得知, 在纳米铁体系中 $\mathrm{pH}$ 的变化是比较 缓慢的, 而纳米镍体系中的 $\mathrm{pH}$ 却升高的很迅速, 即 存在大量酸的不断消耗现象. 为考察反应速率及酸 的消耗的变化, 选择在不同温度下进行试验对比 观察.

图 5 显示的是不同温度 $(298,306,316$ K)下 2,4二氯苯酚的去除以及酸的消耗量情况. 其中, 图 5(a) 和 5(b)分别给出了不同温度下 2,4-二氯苯酚的浓度和 去除率变化情况. 可以看出, 在酸性体系内, 室温下 2,4-二氯苯酚即可在 $5 \mathrm{~h}$ 内达到 $99 \%$ 以上的去除率. 升高温度, 反应速率迅速提高, 并且在相同的反应时 间内的去除率增大. 在温度为 $33^{\circ} \mathrm{C}$ 时, $3 \mathrm{~h}$ 便可完全去 除; $43^{\circ} \mathrm{C}$ 时更是 $30 \mathrm{~min}$ 内达到 $90 \%$ 以上的去除率, $2 \mathrm{~h}$
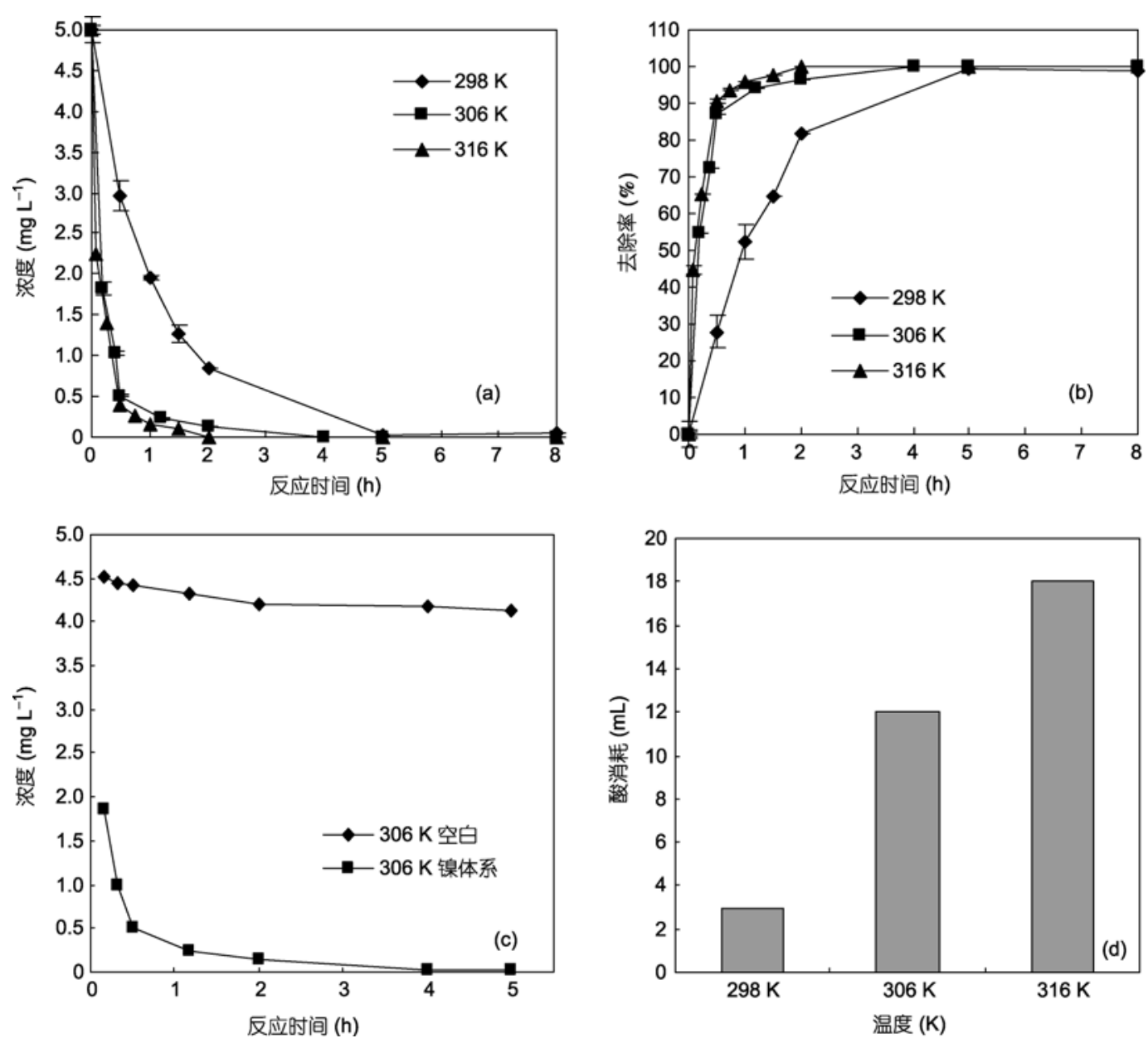

图 5 不同温度条件下, 纳米镍对 2,4-二氯苯酚的脱氯降解去除情况

(a) 浓度变化; (b) 去除率变化; (c) 在温度 $306 \mathrm{~K}$ 条件下, 纳米镍酸性体系和空白酸性体系中 2,4-二氯苯酚浓度变化对照; (d) 用酸量对比 (酸 度调节使用 $1: 200$ 硫酸) 
内便可 100\%去除. 图 5(c)显示的是在 $306 \mathrm{~K}$ 温度下, 酸性体系中纳米镍降解 2,4-二氯苯酚反应过程中, 2,4-二氯苯酚的浓度变化及其相应的空白对照情况. 图 5(d)显示的是不同温度下酸的消耗量, 可看出酸的 消耗量随着温度升高和去除率的提高而增加, 这一 现象说明在酸性体系下, 纳米镍对 2,4-二氯苯酚的降 解过程中, 酸不仅起到调节 $\mathrm{pH}$ 的作用, 更是作为一 种反应物参与了反应. 不同温度下均检测到 2-氯酚、 4-氯酚、氯离子和苯酚生成, 但由于竞争吸附作用, 2氯酚和 4-氯酚检测结果规律性不强, 并且含量较低, 而苯酚含量的变化则规律性较明显, 结果如图 6 所示. 该实验结果表明, 升高温度, 2,4-二氯苯酚在 $\mathrm{Ni}$ 表面 的化学反应加速, 从而整个脱氯过程加快. 同时也表 明, 在酸性体系下, 纳米镍对 2,4-二氯苯酚的去除效 率是很高的.

图 7 给出了在不同温度 $(298,306,316 \mathrm{~K})$ 条件下 酸性体系中纳米镍降解 2,4 -二氯苯酚的 $1 / C-1 / C_{0}$ 与 时间关系图 $(\mathrm{pH}=3)$. 经线性拟合可知, 在酸性体系内 纳米镍对氯代有机物的降解反应为二级反应, 即 $-\mathrm{d} C / C^{2}=k \mathrm{~d} t$, 积分得 $1 / C=k t+1 / C_{0}$, 其中 $C$ 为反应体 系中氯代有机物的浓度, $C_{0}$ 为氯代有机物初始浓度, $t$ 为反应时间, $k$ 为反应速率常数, 亦即曲线的斜率. 3 种不同温度条件下, 其二级反应拟合方程及相关系 数列于表 2 中. 可以看出, 在 3 种不同温度条件下, 其降解速率常数 $k$ 分别为 $0.02,0.2$ 和 $0.3(\mathrm{~g} \mathrm{Lh})^{-1}$. 另 外, 在中性实验体系中, 经拟合得知反应为准一级反 应, 3 种不同温度下的 $k$ 值分别为 $0.0244,0.0379$ 和

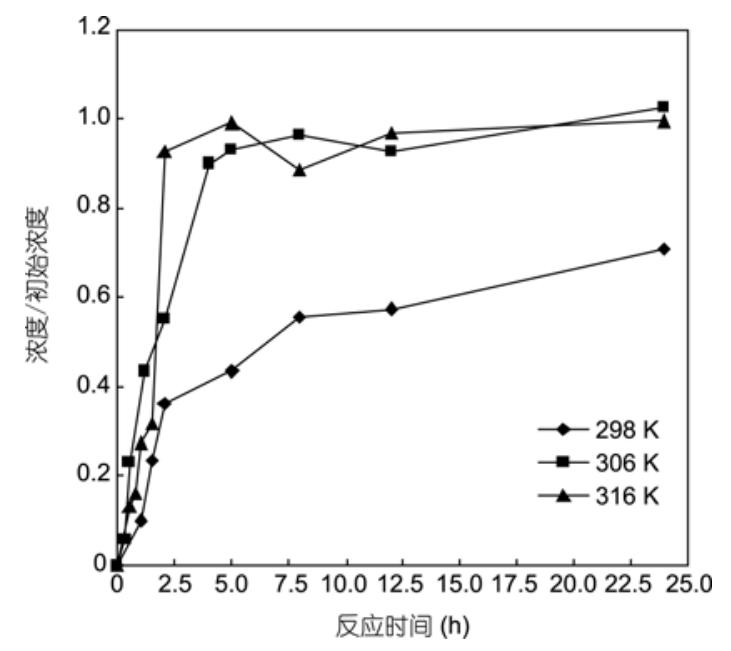

图 6 不同温度条件下, 2,4-二氯苯酚的脱氯降解产物 一苯酚的生成情况

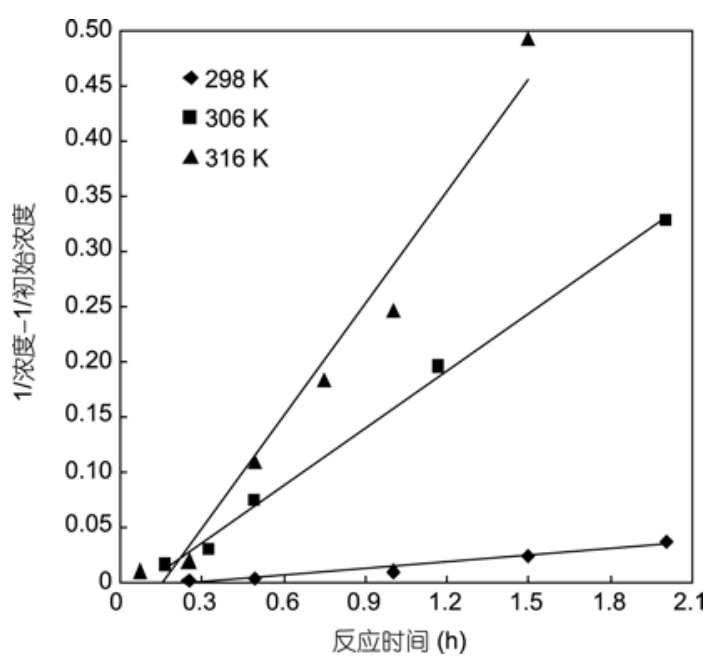

图 7 不同温度条件下, 纳米镍对 2,4 -二氯苯酚的 脱氯降解速率变化情况

表 2 纳米镍对 2,4-二氯苯酚的脱氯降解速率方程 及相关系数

\begin{tabular}{ccc}
\hline 温度 $(\mathrm{K})$ & 拟合方程 & $R^{2}$ \\
\hline 298 & $Y=0.02 x-5 \times 10^{-6}$ & 0.9653 \\
306 & $Y=0.2 x-2 \times 10^{-5}$ & 0.9965 \\
316 & $Y=0.3 x-5 \times 10^{-5}$ & 0.9714 \\
\hline
\end{tabular}

$0.107 \mathrm{~h}^{-1}$, 从而验证了酸作为一种反应物参与了反应 这一结论. 同时还可看出, 升温对反应的促进作用非 常有利, 但温度升高到一定程度, 对反应速率的提高 不再明显，因此反应不宜使用过高温度.

由实验现象和相关文献可知 ${ }^{[20]}$, 纳米镍对 2,4二氯苯酚的脱氯降解主要经过 3 个步骤: (1)纳米镍与 水中的氢离子发生反应, 生成镍离子, 同时产生原子 态 $\mathrm{H}$; (2) 原子态 $\mathrm{H}$ 进一步还原降解 2,4-二氯苯酚; (3) 随着反应的进行和氢离子的消耗, 镍离子与氢氧根 离子形成氢氧化物沉淀并覆盖在金属镍表面, 阻碍 反应进一步发生, 同时也吸附并覆盖住大量目标物, 使其无法完全反应降解. 单独的 $\mathrm{H}_{2}$ 是一种很迟钝的 还原剂, 只有当体系中有催化剂, 或者能够提供某种 催化功能的物质存在的情况下, $\mathrm{H}_{2}$ 的快速还原作用 才成为可能. $\mathrm{Ni}$ 作为常用的加氢催化剂, 其表面对 $\mathrm{H}_{2}$ 有很强的吸附能力, 并将其分解为还原性更强的原 子态 $\mathrm{H}$, 从而加速还原脱氯反应. 2,4-二氯苯酚在纳 米镍上发生脱氯反应的主要原因是吸附氢对其的还 原作用. 在中性体系中, 镍同时充当还原金属和催化 
剂, 而镍与水反应较缓慢, 从而效率较低; 而在酸性 体系中, 大量的 $\mathrm{H}$ 由酸与纳米镍反应提供, 而非依赖 纳米镍与水发生反应, 纳米镍主要起到吸附储 $\mathrm{H}$ 并 催化还原脱氯的作用, 因此反应活性和脱氯效率得 以大大增强. 而酸的存在不仅提供了质子, 还有以下 几种用途: (1)腐蚀金属镍, 加速反应; (2)阻止难溶的 金属氧化物或氢氧化物的形成; (3)抑制纳米金属表 面生成疏松的氧化层对目标物的吸附, 从而促进脱 氯降解反应.

\section{4 纳米镍对 2,4-二氯苯酚降解产物的吸附解吸 情况}

在中性体系的实验过程中, 2,4-二氯苯酚的主要 降解产物苯酚的出现并无明显的规律性, 其主要原 因可能是纳米镍颗粒物对产物的吸附作用所造成. 根据之前的研究结果, 纳米金属对氯酚的降解基本 保留于脱氯阶段, 一般情况下不能发生开环反应, 即 纳米金属对苯酚的去除只有可能存在吸附作用. 为 此, 我们就实验过程中纳米镍对降解产物苯酚的吸 着情况, 做了进一步研究. 实验中苯酚的初始浓度为 $2.8 \mathrm{mg} / \mathrm{L}$, 总体积为 $100 \mathrm{~mL}$.

如图 8 所示, 在中性体系中, 纳米镍对苯酚的吸 附率在 $20 \%$ 35\%之间, 离心后震荡反应可解吸出吸 附量的 20\%左右. 从吸附量上看, 尽管本体系中纳米 镍对苯酚的吸附量不高(少于 $0.1 \mathrm{mg}$ ), 但本实验中选 择的 2,4-二氯苯酚初始浓度为 $5 \mathrm{mg} / \mathrm{L}$, 体积为 $200 \mathrm{~mL}$, 总量为 $1 \mathrm{mg}$, 即使被完全降解产生的苯酚量也只在 $0.56 \mathrm{mg}$ 左右, 而中性体系内纳米镍对 2,4-二氯苯酚
的降解率不高(低于 70\%), 其本身产生的苯酚产物的 量不大, 而且大部分都能够被纳米镍及其表面氧化 物覆盖层所吸附, 从而本体系中纳米镍对苯酚的吸 附作用足以影响其在实验过程中的行为, 导致其苯 酚生成量没有表现出应有的规律性.

\section{3 结论}

(1) 本实验所用羰基纳米镍颗粒粒径为 10 20 nm, BET 比表面积为 $32.40 \mathrm{~m}^{2} / \mathrm{g}$, 与相同工艺制备的纳米 铁粉相比, 其比表面积增加近 4 倍. 受羰基法生产制 备工艺影响, 纳米镍表面存在有一层较薄的氧化层 $(\mathrm{NiO})$, 且其组成成分中还含有一定量的碳元素, 从 而使其对有机物具有一定的吸附性能.

（2）酸洗活化可除去纳米镍表面的氧化层, 从而 提高其对 2,4-二氯苯酚的脱氯降解反应效率, 但在中 性体系中反应时, 由于体系 $\mathrm{pH}$ 值的升高引起新生氧 化物和氢氧化物层在纳米镍表面的包裹覆盖, 从而 导致不完全的脱氯降解反应发生. 反应过程中因消 耗质子需要定期补加酸量, 以维持体系适当的酸度, 酸不仅起到调节 $\mathrm{pH}$ 的作用, 更是作为一种反应物参 与了反应, 从而极大地促进了反应进程.

(3) 在酸性体系 ( $\mathrm{pH}$ 3 4) 中, 纳米镍对 2,4-二氯 苯酚有很高的脱氯降解效率, 可在 $4 \mathrm{~h}$ 内达到 $100 \%$ 的去除率, 并生成大量脱氯降解反应产物苯酚. 实验 证实, 酸性体系中纳米镍对氯代有机物的脱氯降解 反应为二级反应, 其反应速率随温度的升高而升高, 在三种不同温度 $(298,306,316 \mathrm{~K})$ 条件下, 其脱氯降 解反应速率常数 $k$ 分别为 $0.02,0.2$ 和 $0.3(\mathrm{~g} \mathrm{Lh})^{-1}$.

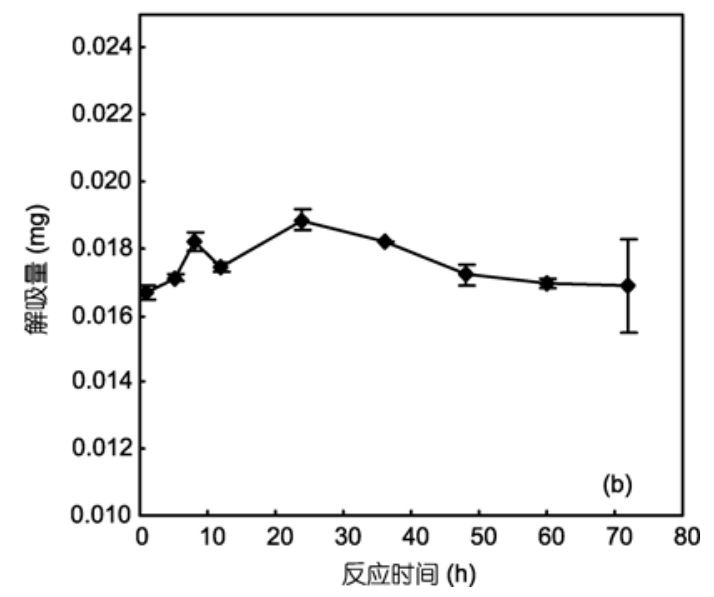

图 8 纳米镍对 2, 4-二氯苯酚的降解产物即苯酚的吸附和解吸情况

(a) 吸附过程; (b) 解吸过程 
致谢＼cjkstart感谢中国科学院生态环境研究中心环境水质学国家重点实验室对本论文实验工作给予的大力支持和热心关怀.

\section{参考文献}

1 Zhang W X, Wang C B, Lien H L. Treatment of chlorinated organic contaminants with nanoscale bimetallic particles. Catalysis Today, 1998, 40: 387-395

2 Ball P, Garwin L. Science at the atomic scale. Nature, 1992, 355: 761-766

3 Chou S, Chang Y, Hwang K Y, et al. Kinetics of reductive denitrification by nanoscale zero-valent iron. Chemosphere, 2000, 41: $1307-1311$

4 Song H, Caraway E. Reduction of chlorinated ethanes by nanosized zero-valent iron: kinetics, pathways and effects of reaction conditions. Environ Sci Technol, 2005, 39: 6237-6245

5 Alessi D S, Zhao H L. Synergistic effect of cationic surfactants on perchloroethylene degradation by zero-valent iron. Environ Sci Technol, 2001, 35: 3713-3717

6 Lowry G V, Johnson K M. Congener-specific dechlorination of dissolved PCBs by microscale and nanoscale zero-valent iron in a water/methanol solution. Environ Sci Technol, 2004, 38: 5208-5216

7 Jovanovic G N, Znidarsic P, Sakrittichai P, et al. Dechlorination of p-chlorophenol in a microreactor with bimetallic Pd/Fe catalys . Ind Eng Chem Res, 2005, 44: 5099-5106

8 Dombek T, Davis D, Stine J, et al. Degradation of terbutylazine(2-chloro-4-ethylamino-6-terbutylamino-1, 3, 5-triazine) deisopropyl atrazine (2-amino-4-chloro-6-ethylamino-1,3,5-triazine) and chlorinated dimethoxy triazine (2-chloro-4, 6-dimethoxy-1,3,5-triazine) by zero valent iron and electrochemical reduction. Environ Poll, 2004, 129: 267-275

9 Keum Y S, Li Q X. Reductive debromination of polybrominated diphenyl ethers by zerovalent iron. Environ Sci Technol, 2005, 39: $2282-2286$

10 Masciangioli T, Zhang W X. Environmental technologies at the nanoscale. Environ Sci Technol, 2003, 37: 102A-108A

11 Zhang W X. Nanoscale iron particles for environmental remediation: an overview. J Nanopart Res, 2003, 5: 323-332

12 Hou C F, Ge X P, Zhou Y M. Characterization of nanoscale iron and its degradation of 2, 4-dichlorophenol. Chinese Sci Bull, 2010, 55: 350-357

13 Kim Y H, Carraway E R. Dechlorination of pentachlorophenol by zero valent iron and modified zero valent irons. Environ Sci Technol, 2000, 34: 2014-2017

14 Rong M Z, Zhang M Q, Wang H B, et al. Surface modification of magnetic metal nanoparticles and its influence on the performance of polymer composites. J Poly Sci (Part B): Poly Phys, 2003, 41: 1070-1084

15 屈子梅. 羰基法生产纳米镍. 粉末冶金工业, 2003, 13: 16-19

16 Kung K S, McBride M B. Bonding of chlorophenol on iron and aluminum-oxides. Environ Sci Technol, 1991, 25: 702-709

17 Burris D R, Campbell T J, Manoranjan V S. Sorption of trichloroethylene and tetrachloroethylene in a batch reactive metallic iron-water system. Environ Sci Technol, 1995, 29: 2850-2855

18 Allen-King R M, Halket R M, Burris D R. Reductive transformation and sorption of cis- and trans-1, 2-dichlorothene in a metallic iron/water system. Environ Toxicol Chem, 1997, 16: 424-429

19 Deng B, Campbell T J, Burris D R. Reduction of vinyl chloride in metallic iron-water systems. Environ Sci Technol, 1997, 31: 1185-1190

20 Liu Y H, Yang F L, Yue P L, et al. Catalytic dechlorination of chlorophenols in water by palladium/iron. Wat Res, 2001, 35: 1887-1890 